\title{
CRITICAL POINT APPROACHES TO QUASILINEAR SECOND ORDER DIFFERENTIAL EQUATIONS DEPENDING ON A PARAMETER
}

\author{
Shapour HeidarkHani - JohnNy Henderson
}

\begin{abstract}
In this paper, we make application of some three-critical points results to establish the existence of at least three solutions for a boundary value problem for the quasilinear second order differential equation on a compact interval $[a, b] \subset \mathbb{R}$,
\end{abstract}

$$
\left\{\begin{array}{l}
-u^{\prime \prime}=(\lambda f(x, u)+g(x, u)) h\left(x, u^{\prime}\right) \quad \text { in }(a, b) \\
u(a)=u(b)=0
\end{array}\right.
$$

under appropriate hypotheses. We exhibit the existence of at least three (weak) solutions.

\section{Introduction}

There is a wide literature that deals with the existence of multiple solutions to two-point boundary value problems. Conditions that guarantee the existence of multiple solutions to differential equations are of interest because physical processes described by differential equations can exhibit more than one solution. For example, certain chemical reactions in tubular reactors can be mathematically described by a nonlinear two-point boundary value problem with the interest in seeing if multiple steady-states to the problem exist. For a treatment of chemical

2010 Mathematics Subject Classification. 34B15, 47J10.

Key words and phrases. Dirichlet problem, critical point, three solutions, variational methods. 
reactor theory and multiple solutions see $[2$, Section 7$]$ and references therein, and for additional approaches to the existence of multiple solutions to boundary value problems, see [5], [22], [23] and references therein.

It is worth mentioning that in a very interesting paper [29], Ricceri obtained a three-critical points theorem and in [28] gave a general version of the theorem to extend the results for a class of more extensive equations. Many works have been carried out in discussing the existence of multiple solutions for BVPs by these Ricceri's results, and we cite, for example, the papers [1], [7], [17], [21] and [25]. In [7] Bonanno illustrated the Ricceri's three-critical points theorem [29] for the existence of three (classical) solutions in the the Sobolev space $W_{0}^{1,2}([0,1])$ for the following two-point boundary value problem

$$
\left\{\begin{array}{l}
u^{\prime \prime}+\lambda f(u)=0 \quad \text { in }(0,1), \\
u(0)=u(1)=0,
\end{array}\right.
$$

where $f: \mathbb{R} \rightarrow \mathbb{R}$ is a continuous function and $\lambda$ is a positive parameter, while in [17] Candito extended the results to the following nonautonomous two-point boundary value problem

$$
\left\{\begin{array}{l}
u^{\prime \prime}+\lambda f(x, u)=0 \quad \text { in }(a, b) \\
u(a)=u(b)=0
\end{array}\right.
$$

where $f:[a, b] \times \mathbb{R} \rightarrow \mathbb{R}$ is a continuous function and $\lambda$ is a positive parameter. Livrea in [25] extended the results of [7], [17], and established multiplicity results for the following ordinary Dirichlet problem

$$
\left\{\begin{array}{l}
u^{\prime \prime}+\lambda f(x, u) h\left(u^{\prime}\right)=0 \quad \text { in }(a, b), \\
u(0)=u(1)=0,
\end{array}\right.
$$

where $f:[0,1] \times \mathbb{R} \rightarrow \mathbb{R}$ and $h: \mathbb{R} \rightarrow] 0,+\infty[$ are two continuous functions and $\lambda>0$.

In particular, based on Ricceri's three-critical points theorem, revisited in [27], the paper [21] deals with the existence of at least three (weak) solutions for the following double-eigenvalue problem with eigenvalues $\lambda>0$ and $\mu>0$ on a bounded interval $(a, b)$ in $\mathbb{R}(a<b)$,

$$
\left\{\begin{array}{l}
u^{\prime \prime}+(\lambda f(t, u)+g(u)) h\left(t, u^{\prime}\right)=\mu p(t, u) h\left(t, u^{\prime}\right) \text { in }(a, b), \\
u(a)=u(b)=0,
\end{array}\right.
$$

where $f:[a, b] \times \mathbb{R} \rightarrow \mathbb{R}$ is a continuous function, $g: \mathbb{R} \rightarrow \mathbb{R}$ is a Lipschitz continuous function with $g(0)=0, h:[a, b] \times \mathbb{R} \rightarrow \mathbb{R}$ is a bounded and continuous function, with $m:=\inf h>0$, and $p:[a, b] \times \mathbb{R} \rightarrow \mathbb{R}$ is an $L^{1}$-Carathéodory function. 
Consider the following quasilinear two-point boundary value problem

$$
\left\{\begin{array}{l}
-u^{\prime \prime}=(\lambda f(x, u)+g(x, u)) h\left(x, u^{\prime}\right) \quad \text { in }(a, b), \\
u(a)=u(b)=0,
\end{array}\right.
$$

where $[a, b] \subset \mathbb{R}$ is a compact interval, $f:[a, b] \times \mathbb{R} \rightarrow \mathbb{R}$ is an $L^{1}$-Carathéodory function, $g:[a, b] \times \mathbb{R} \rightarrow \mathbb{R}$ is a continuous function such that there exists a constant $L \geq 0$ provided

$$
\left|g\left(\cdot, t_{1}\right)-g\left(\cdot, t_{2}\right)\right| \leq L\left|t_{1}-t_{2}\right|
$$

for all $t_{1}, t_{2} \in \mathbb{R}$ satisfying $\left.g(\cdot, 0)=0, h:[a, b] \times \mathbb{R} \rightarrow\right] 0,+\infty[$ is a bounded and continuous function, with $m:=\inf h>0$, and $\lambda$ is a positive parameter.

Employing, two critical points theorems (Theorems 1.2 and 1.3), we establish the existence of at least three distinct (weak) solutions in $W_{0}^{1,2}([a, b])$ to the problem (1.1) (Theorems 2.1 and 2.2).

We mean by a (weak) solution of problem (1.1), any $u \in W_{0}^{1,2}([a, b])$ such that

$\int_{a}^{b}\left[\left(\int_{0}^{u^{\prime}(x)} \frac{1}{h(x, \tau)} d \tau\right) v^{\prime}(x)-g(x, u(x)) v(x)\right] d x-\lambda \int_{a}^{b} f(x, u(x)) v(x) d x=0$ for every $v \in W_{0}^{1,2}([a, b])$.

A special case of Theorem 2.2 is the following theorem.

THEOREM 1.1. Let $f: \mathbb{R} \rightarrow \mathbb{R}$ be a continuous function and $h: \mathbb{R} \rightarrow] 0,+\infty[$ be a bounded and continuous function, with $m:=\inf h>0$. Put $F(t)=\int_{0}^{t} f(\xi) d \xi$ for each $t \in \mathbb{R}$. Assume that $F(d)>0$ for some $d>0$ and $F(\xi) \geq 0$ in $[0, d]$ and

$$
\liminf _{\xi \rightarrow 0} \frac{F(\xi)}{\xi^{2}}=\limsup _{\xi \rightarrow+\infty} \frac{F(\xi)}{\xi^{2}}=0 .
$$

Then, there is $\lambda^{*}>0$ such that for each $\lambda>\lambda^{*}$ the problem

$$
\left\{\begin{array}{l}
-u^{\prime \prime}=\lambda f(u) h\left(u^{\prime}\right) \quad \text { in }(a, b), \\
u(a)=u(b)=0,
\end{array}\right.
$$

admits at least three classical solutions.

Actually, our main results, Theorems 2.1 and 2.2, assure the existence of three solutions to the problem (1.1) under appropriate hypotheses involving a test function $w$, while in Corollaries 2.3, 2.4, 2.6 and 2.7 a convenient choice of $w$ makes the assumptions clearer, if less general.

Our analyses are based on the following three-critical points theorems (see also [6], [26]-[29] for related results) to transfer the existence of three solutions of the problem (1.1) into the existence of critical points of the Euler functional; in the first one the coercivity of the functional $\Phi-\lambda \Psi$ is required, while in the second one a suitable sign hypothesis is assumed. 
Theorem 1.2 ([13, Theorem 2.6]). Let $X$ be a reflexive real Banach space, let $\Phi: X \rightarrow \mathbb{R}$ be a sequentially weakly lower semicontinuous, coercive and continuously Gâteaux differentiable whose Gâteaux derivative admits a continuous inverse on $X^{*}$, and let $\Psi: X \longrightarrow \mathbb{R}$ be a sequentially weakly upper semicontinuous and continuously Gâteaux differentiable functional whose Gâteaux derivative is compact. Assume that there exist $r \in \mathbb{R}$ and $u_{1} \in X$ with $0<r<\Phi\left(u_{1}\right)$, such that:

(a) $\sup _{\left.\left.u \in \Phi^{-1}(]-\infty, r\right]\right)} \Psi(u)<r \frac{\Psi\left(u_{1}\right)}{\Phi\left(u_{1}\right)}$,

(b) for each $\left.\lambda \in \Lambda_{r}:=\right] \frac{\Phi\left(u_{1}\right)}{\Psi\left(u_{1}\right)}, \frac{r}{\sup _{\left.\left.u \in \Phi^{-1}(]-\infty, r\right]\right)} \Psi(u)}[$ the functional $\Phi-\lambda \Psi$ is coercive.

Then, for each $\lambda \in \Lambda_{r}$ the functional $\Phi-\lambda \Psi$ has at least three distinct critical points in $X$.

Theorem 1.3 ([8, Theorem 3.3], see also [3]). Let $X$ be a reflexive real Banach space, $\Phi: X \rightarrow \mathbb{R}$ be a convex, coercive and continuously Gâteaux differentiable functional whose derivative admits a continuous inverse on $X^{*}, \Psi: X \rightarrow \mathbb{R}$ be a continuously Gâteaux differentiable functional whose derivative is compact, such that

(a) $\inf _{X} \Phi=\Phi(0)=\Psi(0)=0$;

(b) for each $\lambda>0$ and for every $u_{1}, u_{2}$ which are local minimum for the functional $\Phi-\lambda \Psi$ and such that $\Psi\left(u_{1}\right) \geq 0$ and $\Psi\left(u_{2}\right) \geq 0$, one has

$$
\inf _{s \in[0,1]} \Psi\left(s u_{1}+(1-s) u_{2}\right) \geq 0 .
$$

Assume that there are two positive constants $r_{1}, r_{2}$ and $\bar{v} \in X$, with $2 r_{1}<$ $\Phi(\bar{v})<r_{2} / 2$, such that

$$
\begin{aligned}
& \sup _{\frac{u \in \Phi^{-1}(]-\infty, r_{1}[)}{r_{1}} \Psi(u)}<\frac{2}{3} \frac{\Psi(\bar{v})}{\Phi(\bar{v})} \\
& \sup _{\frac{u \in \Phi^{-1}(]-\infty, r_{2}[)}{r_{2}} \Psi(u)}<\frac{1}{3} \frac{\Psi(\bar{v})}{\Phi(\bar{v})} .
\end{aligned}
$$

Then, for each

$$
\lambda \in] \frac{3}{2} \frac{\Phi(\bar{v})}{\Psi(\bar{v})}, \min \left\{\frac{r_{1}}{\sup _{u \in \Phi^{-1}(]-\infty, r_{1}[)} \Psi(u)}, \frac{r_{2} / 2}{\sup _{u \in \Phi^{-1}(]-\infty, r_{2}[)} \Psi(u)}\right\}[,
$$

the functional $\Phi-\lambda \Psi$ has at least three distinct critical points which lie in $\Phi^{-1}(]-\infty, r_{2}[)$. 
For other basic notations and definitions, and for a thorough account on the subject, we refer the reader to [9]-[12], [14], [15], [18]-[20], [24].

The outline of the paper is organized as follows: in the forthcoming section, we shall present the statements of our results, proofs of the corollaries and an example to illustrate the results. Section 3 consists the proofs of our main results.

We recall that a function $f:[a, b] \times \mathbb{R} \rightarrow \mathbb{R}$ is said to be $L^{1}$-Carathéodory if

(a) $x \rightarrow f(x, t)$ is measurable for every $t \in \mathbb{R}$,

(b) $t \rightarrow f(x, t)$ is continuous for almost every $x \in[a, b]$,

(c) for every $\varrho>0$ there exists a function $l_{\varrho} \in L^{1}([a, b])$ such that

$$
\sup _{|t| \leq \varrho}|f(x, t)| \leq l_{\varrho}(x) \quad \text { for a.e. } x \in[a, b] .
$$

\section{Main results}

Let $f:[a, b] \times \mathbb{R} \rightarrow \mathbb{R}$ be an $L^{1}$-Carathéodory function, $g:[a, b] \times \mathbb{R} \rightarrow \mathbb{R}$ be a continuous function such that there exists a constant $L \geq 0$ provided

$$
\left|g\left(\cdot, t_{1}\right)-g\left(\cdot, t_{2}\right)\right| \leq L\left|t_{1}-t_{2}\right| \text { for all } t_{1}, t_{2} \in \mathbb{R}
$$

satisfying $g(\cdot, 0)=0$. Let $h:[a, b] \times \mathbb{R} \rightarrow] 0,+\infty[$ be a bounded and continuous function with $m:=\inf h>0$. Denote $M:=\sup h$ and suppose that the constant $L \geq 0$ satisfies $L M(b-a)^{2}<4$.

We introduce the functions $F:[a, b] \times \mathbb{R} \rightarrow \mathbb{R}, H:[a, b] \times \mathbb{R} \rightarrow \mathbb{R}$ and $G:[a, b] \times$ $\mathbb{R} \rightarrow \mathbb{R}$ respectively, as follows

$$
\begin{array}{lll}
F(x, t) & =\int_{0}^{t} f(x, \xi) d \xi & \text { for all }(x, t) \in[a, b] \times \mathbb{R}, \\
H(x, t)=\int_{0}^{t} \int_{0}^{\tau} \frac{1}{h(x, \delta)} d \delta d \tau & \text { for all }(x, t) \in[a, b] \times \mathbb{R}, \\
G(x, t)=-\int_{0}^{t} g(x, \xi) d \xi & \text { for all }(x, t) \in[a, b] \times \mathbb{R} .
\end{array}
$$

We now formulate our main results.

THEOREM 2.1. Assume that there exist a positive constant $r$ and a function $w \in W_{0}^{1,2}([a, b])$ such that:

(a) $\int_{a}^{b}\left[G(x, w(x))+H\left(x, w^{\prime}(x)\right)\right] d x>r$,

(b) $\frac{1}{r} \int_{a}^{b} \sup _{t \in\left[-\sqrt{\frac{2 M(b-a) r}{4-L M(b-a)^{2}}}, \sqrt{\frac{2 M(b-a) r}{4-L M(b-a)^{2}}}\right]} F(x, t) d x$

$$
<\int_{a}^{b} F(x, w(x)) d x / \int_{a}^{b}\left[G(x, w(x))+H\left(x, w^{\prime}(x)\right)\right] d x,
$$


(c) $\limsup _{|t| \rightarrow+\infty} \frac{F(x, t)}{t^{2}}<\frac{4-L M(b-a)^{2}}{2 M(b-a)^{2} r}$

uniformly respect to $x \in[a, b]$.

$$
\int_{a}^{b} \sup _{t \in\left[-\sqrt{\frac{2 M(b-a) r}{4-L M(b-a)^{2}}}, \sqrt{\frac{2 M(b-a) r}{4-L M(b-a)^{2}}}\right]} F(x, t) d x
$$

Then, for each

$$
\begin{aligned}
& \left.\lambda \in \Lambda_{1}:=\right] \int_{a}^{b}\left[G(x, w(x))+H\left(x, w^{\prime}(x)\right)\right] d x / \int_{a}^{b} F(x, w(x)) d x \\
& r / \int_{a}^{b} \sup _{t \in\left[-\sqrt{\frac{2 M(b-a) r}{4-L M(b-a)^{2}}}, \sqrt{\frac{2 M(b-a) r}{4-L M(b-a)^{2}}}\right]} F(x, t) d x[
\end{aligned}
$$

the problem (1.1) admits at least three distinct weak solutions in $W_{0}^{1,2}([a, b])$.

TheOREM 2.2. Suppose that $f:[0,1] \times \mathbb{R} \rightarrow \mathbb{R}$ satisfies the condition $f(x, t) \geq 0$ for all $x \in[0,1]$ and $t \geq 0$. Assume that there exist a function $w \in W_{0}^{1,2}([a, b])$ and two positive constants $r_{1}$ and $r_{2}$ with

$$
2 r_{1}<\int_{a}^{b}\left[G(x, w(x))+H\left(x, w^{\prime}(x)\right)\right] d x<\frac{r_{2}}{2}
$$

such that:

$$
\text { (a) } \begin{aligned}
\frac{1}{r_{1}} \int_{a}^{b} & \sup _{t \in\left[-\sqrt{\frac{2 M(b-a) r_{1}}{4-L M(b-a)^{2}}}, \sqrt{\frac{2 M(b-a) r_{1}}{4-L M(b-a)^{2}}}\right.}^{b} \\
& <\frac{2}{3} \int_{0}^{1} F(x, t) d x
\end{aligned}
$$

(b) $\frac{1}{r_{2}} \int_{a}^{b} \sup _{t \in\left[-\sqrt{\frac{2 M(b-a) r_{2}}{4-L M(b-a)^{2}}}, \sqrt{\frac{2 M(b-a) r_{2}}{4-L M(b-a)^{2}}}\right]} F(x, t) d x$

$$
<\frac{1}{3} \int_{0}^{1} F(x, w(x)) d x / \int_{a}^{b}\left[G(x, w(x))+H\left(x, w^{\prime}(x)\right)\right] d x .
$$

Then, for each

$$
\lambda \in] \frac{3}{2} \int_{a}^{b}\left[G(x, w(x))+H\left(x, w^{\prime}(x)\right)\right] d x / \int_{0}^{1} F(x, w(x)) d x, \Theta_{1}[,
$$


where

$$
\begin{aligned}
& \Theta_{1}:=\min \left\{r_{1} / \int_{a}^{b} \sup _{t \in\left[-\sqrt{\frac{2 M(b-a) r_{1}}{4-L M(b-a)^{2}}}, \sqrt{\frac{2 M(b-a) r_{1}}{4-L M(b-a)^{2}}}\right]} F(x, t) d x,\right. \\
& \left.\frac{r_{2}}{2} / \int_{a}^{b} \sup _{t \in\left[-\sqrt{\frac{2 M(b-a) r_{2}}{4-L M(b-a)^{2}}}, \sqrt{\frac{2 M(b-a) r_{2}}{4-L M(b-a)^{2}}}\right]} F(x, t) d x\right\} \text {, }
\end{aligned}
$$

the problem (1.1) admits at least three non-negative weak solutions $v^{1}, v^{2}, v^{3}$ such that

$$
\left|v^{j}(x)\right|<\sqrt{\frac{2 M(b-a) r_{2}}{4-L M(b-a)^{2}}} \quad \text { for each } x \in[a, b], j=1,2,3 .
$$

Let us first give particular consequences of Theorems 2.1 and 2.2for a fixed test function $w$.

Corollary 2.3. Assume that there exist four positive constants $c, d, \mu$ and $\nu$ with $\mu+\nu<b-a$ such that:

$$
\begin{aligned}
& \text { (a) } K_{1}:=\int_{a}^{a+\mu}\left[G\left(x, \frac{d}{\mu}(x-a)\right)+H\left(x, \frac{d}{\mu}\right)\right] d x+\int_{a+\mu}^{b-\nu} G(x, d) d x \\
& +\int_{b-\nu}^{b}\left[G\left(x, \frac{d}{\nu}(b-x)\right)+H\left(x,-\frac{d}{\nu}\right)\right] d x>\frac{2 c^{2}}{M(b-a)}, \\
& \text { (b) } \frac{M(b-a)}{2 c^{2}} \int_{a}^{b} \sup _{t \in\left[-\sqrt{\frac{4 c^{2}}{4-L M(b-a)^{2}}}, \sqrt{\frac{4 c^{2}}{4-L M(b-a)^{2}}}\right]} F(x, t) d x<\frac{K_{2}}{K_{1}} \\
& \text { where } \\
& K_{2}:=\int_{a}^{a+\mu} F\left(x, \frac{d}{\mu}(x-a)\right) d x+\int_{a+\mu}^{b-\nu} F(x, d) d x+\int_{b-\nu}^{b} F\left(x, \frac{d}{\nu}(b-x)\right) d x \\
& \text { (c) } \limsup _{|t| \rightarrow+\infty} \frac{F(x, t)}{t^{2}} \\
& <\frac{4-L M(b-a)^{2}}{4(b-a) c^{2}} \int_{a}^{b} \sup _{t \in\left[-\sqrt{\frac{4 c^{2}}{4-L M(b-a)^{2}}}, \sqrt{\frac{4 c^{2}}{4-L M(b-a)^{2}}}\right]} F(x, t) d x
\end{aligned}
$$

uniformly with respect to $x \in[a, b]$.

Then, for each

$$
\left.\lambda \in \Lambda_{2}:=\right] \frac{K_{1}}{K_{2}}, 2 c^{2} / M(b-a) \int_{a}^{b} \sup _{t \in\left[-\sqrt{\frac{4 c^{2}}{4-L M(b-a)^{2}}}, \sqrt{\frac{4 c^{2}}{4-L M(b-a)^{2}}}\right]} F(x, t) d x[
$$


the problem (1.1) admits at least three distinct weak solutions in $W_{0}^{1,2}([a, b])$.

Proof. Owing to our hypotheses, we observe that all the assumptions of Theorem 2.1 are satisfied by choosing

$$
w(x)= \begin{cases}\frac{d}{\mu}(x-a) & \text { if } a \leq x<a+\mu \\ d & \text { if } a+\mu \leq x \leq b-\nu, \\ \frac{d}{\nu}(b-x) & \text { if } b-\nu<x \leq b,\end{cases}
$$

and $r=2 c^{2} /(M(b-a))$. Hence, the conclusion follows directly from Theorem 2.1 .

Corollary 2.4. Let $f:[a, b] \times \mathbb{R} \rightarrow \mathbb{R}$ satisfies the condition $f(x, t) \geq 0$ for all $x \in[a, b]$ and $t \geq 0$. Assume that there exist five positive constants $c_{1}, c_{2}, d$, $\mu$ and $\nu$ with $\mu+\nu<b-a$ such that:

(a) $\frac{4 c_{1}^{2}}{M(b-a)}<K_{1}<\frac{c_{2}^{2}}{M(b-a)}$

(b) $\frac{M(b-a)}{2 c_{1}^{2}} \int_{a}^{b} \sup _{t \in\left[-\sqrt{\frac{4 c_{1}^{2}}{4-L M(b-a)^{2}}}, \sqrt{\frac{4 c_{1}^{2}}{4-L M(b-a)^{2}}}\right]} F(x, t) d x<\frac{2}{3} \frac{K_{2}}{K_{1}}$,

(c) $\frac{M(b-a)}{2 c_{2}^{2}} \int_{a}^{b} \sup _{t \in\left[-\sqrt{\frac{4 c_{2}^{2}}{4-L M(b-a)^{2}}}, \sqrt{\frac{4 c_{2}^{2}}{4-L M(b-a)^{2}}}\right]} F(x, t) d x<\frac{1}{3} \frac{K_{2}}{K_{1}}$,

where $K_{1}$ and $K_{2}$ are given as in assumptions (a) and (b) in Corollary 2.3 , respectively.

Then, for each $\lambda \in] 3 K_{1} /\left(2 K_{2}\right), \Theta_{2}[$, where

$$
\begin{aligned}
\Theta_{2}:=\min \left\{\frac{2 c_{1}^{2}}{M(b-a)} / \int_{a}^{b} \sup _{t \in\left[-\sqrt{\frac{4 c_{1}^{2}}{4-L M(b-a)^{2}}}, \sqrt{\frac{4 c_{1}^{2}}{4-L M(b-a)^{2}}}\right]} F(x, t) d x,\right. \\
\left.\frac{c_{2}^{2}}{M(b-a)} / \int_{a}^{b} \sup _{t \in\left[-\sqrt{\frac{4 c_{2}^{2}}{4-L M(b-a)^{2}}}, \sqrt{\frac{4 c_{2}^{2}}{4-L M(b-a)^{2}}}\right]} F(x, t) d x\right\},
\end{aligned}
$$

the problem (1.1) admits at least three non-negative weak solutions $v^{1}, v^{2}, v^{3}$ such that

$$
\left|v^{j}(x)\right|<\sqrt{\frac{4 c_{2}^{2}}{4-L M(b-a)^{2}}} \quad \text { for each } x \in[a, b], j=1,2,3 .
$$

Proof. Proceeding the same way as in the proof of Corollary 2.3, we achieve the stated assertion by applying Theorem 2.2 with $w$ as given in $(2.2), r_{1}=$ $2 c_{1}^{2} /(M(b-a))$ and $r_{2}=2 c_{1}^{2} /(M(b-a))$. 
REMARK 2.5. Other candidates for the test function $w$ in (2.2) can be considered for other versions of the statement. For example, in (2.2), one can choose $\mu=\nu=(b-a) / 4$ which is a particular case.

Let $f$ and $F$ be as before, and let $g: \mathbb{R} \rightarrow \mathbb{R}$ be a Lipschitz continuous function with the Lipschitz constant $L \geq 0$, i.e.

$$
\left|g\left(t_{1}\right)-g\left(t_{2}\right)\right| \leq L\left|t_{1}-t_{2}\right| \text { for all } t_{1}, t_{2} \in \mathbb{R},
$$

satisfying $g(0)=0$. Let $h: \mathbb{R} \rightarrow] 0,+\infty[$ be a bounded and continuous function with $m:=\inf h>0$. Denote $M:=\sup h$ and suppose that the Lipschitz constant $L \geq 0$ satisfies $L M(b-a)^{2}<4$. We introduce the functions $H: \mathbb{R} \rightarrow \mathbb{R}$ and $G: \mathbb{R} \rightarrow \mathbb{R}$ respectively, as follows

$$
\begin{array}{ll}
H(t)=\int_{0}^{t} \int_{0}^{\tau} \frac{1}{h(\delta)} d \delta d \tau & \text { for all } t \in \mathbb{R}, \\
G(t)=-\int_{0}^{t} g(\xi) d \xi & \text { for all } t \in \mathbb{R} .
\end{array}
$$

Then, we have the following results as consequences of Corollaries 2.3 and 2.4, respectively:

Corollary 2.6. Assume that there exist four positive constants $c, d, \mu$ and $\nu$ with $\mu+\nu<b-a$ such that Assumption (c)

(a) $F(x, t) \geq 0$ for each $(x, t) \in([a, a+\mu] \cup[b-\nu, b]) \times[0, d]$,

(b) $K_{3}:=\frac{\mu+\nu}{d} \int_{0}^{d} G(t) d t+(b-a-\mu-\nu) G(d)+\mu H\left(\frac{d}{\mu}\right)+\nu H\left(-\frac{d}{\nu}\right)$

$$
>\frac{2 c^{2}}{M(b-a)}
$$

(c) $\frac{M(b-a)}{2 c^{2}} \int_{a}^{b} \sup _{t \in\left[-\sqrt{\frac{4 c^{2}}{4-L M(b-a)^{2}}}, \sqrt{\frac{4 c^{2}}{4-L M(b-a)^{2}}}\right]} F(x, t) d x$

$$
<\frac{1}{K_{3}} \int_{a+\mu}^{b-\nu} F(x, d) d x .
$$

Then, for each

$$
\begin{aligned}
& \left.\lambda \in \Lambda_{3}:=\right] K_{3} / \int_{a+\mu}^{b-\nu} F(x, d) d x, \\
& 2 c^{2} / M(b-a) \int_{a}^{b} \sup _{t \in\left[-\sqrt{\frac{4 c^{2}}{4-L M(b-a)^{2}}}, \sqrt{\frac{4 c^{2}}{4-L M(b-a)^{2}}}\right]} F(x, t) d x[
\end{aligned}
$$

the problem

$$
\left\{\begin{array}{l}
-u^{\prime \prime}=(\lambda f(x, u)+g(u)) h\left(u^{\prime}\right) \quad \text { in }(a, b), \\
u(a)=u(b)=0
\end{array}\right.
$$


admits at least three distinct weak solutions in $W_{0}^{1,2}([a, b])$.

Proof. Since $0 \leq w(x) \leq d$ for each $x \in[a, b]$ where $w$ is given as in (2.2), the condition (a) ensures that

$$
\int_{a}^{a+\mu} F(x, w(x)) d x+\int_{b-\nu}^{b} F(x, w(x)) d x \geq 0 .
$$

So,

$$
\int_{a}^{b} F(x, w(x)) d x \geq \int_{a+\mu}^{b-\nu} F(x, d) d x .
$$

Hence, with $g(x, t)=g(t)$ and $h(x, t)=h(t)$ for all $(x, t) \in[a, b] \times \mathbb{R}$, from Corollary 2.3 we have the conclusion.

Corollary 2.7. Let $f:[a, b] \times \mathbb{R} \rightarrow \mathbb{R}$ satisfies the condition $f(x, t) \geq 0$ for all $x \in[a, b]$ and $t \geq 0$. Assume that there exist five positive constants $c_{1}, c_{2}, d$, $\mu$ and $\nu$ with $\mu+\nu<b-a$ such that
(a) $\frac{4 c_{1}^{2}}{M(b-a)}<K_{3}<\frac{c_{2}^{2}}{M(b-a)}$,
(b) $\frac{M(b-a)}{2 c_{1}^{2}} \int_{a}^{b} \sup _{t \in\left[-\sqrt{\frac{4 c_{1}^{2}}{4-L M(b-a)^{2}}}, \sqrt{\frac{4 c_{1}^{2}}{4-L M(b-a)^{2}}}\right]} F(x, t) d x$
(c) $\frac{M(b-a)}{2 c_{2}^{2}} \int_{a}^{b} \sup _{t \in\left[-\sqrt{\frac{4 c_{2}^{2}}{4-L M(b-a)^{2}}}, \sqrt{\frac{4 c_{2}^{2}}{4-L M(b-a)^{2}}}\right]} F(x, t) d x$

$$
<\frac{2}{3 K_{3}} \int_{a+\mu}^{b-\nu} F(x, d) d x,
$$

where $K_{3}$ is given as in Assumptions (b) in Corollary 2.6.

Then, for each

$$
\lambda \in] \frac{3}{2} K_{3} / \int_{a+\mu}^{b-\nu} F(x, d) d x, \Theta_{3}[
$$

where

$$
\begin{array}{r}
\Theta_{3}:=\min \left\{\frac{2 c_{1}^{2}}{M(b-a)} / \int_{a}^{b} \sup _{t \in\left[-\sqrt{\frac{4 c_{1}^{2}}{4-L M(b-a)^{2}}}, \sqrt{\frac{4 c_{1}^{2}}{4-L M(b-a)^{2}}}\right]} F(x, t) d x,\right. \\
\left.\frac{c_{2}^{2}}{M(b-a)} / \int_{a}^{b} \sup _{t \in\left[-\sqrt{\frac{4 c_{2}^{2}}{4-L M(b-a)^{2}}}, \sqrt{\frac{4 c_{2}^{2}}{4-L M(b-a)^{2}}}\right]} F(x, t) d x\right\},
\end{array}
$$


the problem (2.3) admits at least three non-negative weak solutions $v^{1}, v^{2}, v^{3}$ such that

$$
\left|v^{j}(x)\right|<\sqrt{\frac{4 c_{2}^{2}}{4-L M(b-a)^{2}}} \quad \text { for each } x \in[a, b], j=1,2,3 .
$$

PROof. Following the same way as in the proof of Corollary 2.6, we achieve the stated assertion by applying Corollary 2.4 .

Let us now present an application of Corollary 2.6.

Example 2.8. Let $[a, b]=[0,1]$. Choose $c=1, d=2, \mu=\nu=1 / 4$, $f(x, t)=\left(t^{+}\right)^{\gamma-1} e^{x-t^{+}}\left(\gamma-t^{+}\right), g(t)=t^{+}$for all $x \in[0,1]$ and $t \in \mathbb{R}$ where $t^{+}=\max \{t, 0\}$ and

$$
\gamma>\log _{2 \sqrt{3} / 3} \frac{89}{6} \frac{e^{-\sqrt{3}}\left(e^{3}-e^{2}\right)}{e^{3 / 4}-e^{1 / 4}}
$$

is a real number, and $h(t)=1 /(2+\sin t)$ for each $t \in \mathbb{R}$. Note that

$$
\begin{aligned}
& F(x, t)= \begin{cases}0 & \text { for all }(x, t) \in[0,1] \times \mathbb{R}^{-}, \\
t^{\gamma} e^{x-t} & \text { for all }(x, t) \in[0,1] \times\left(\mathbb{R}^{+} \cup\{0\}\right),\end{cases} \\
& H(t)=t^{2}+t-\sin t \quad \text { for all } t \in \mathbb{R}, \\
& G(t)= \begin{cases}0 & \text { for all } t \in \mathbb{R}^{-}, \\
-\frac{t^{2}}{2} & \text { for all } t \in \mathbb{R}^{+} \cup\{0\},\end{cases}
\end{aligned}
$$

and $L=M=1$, clearly assumption (a) in Corollary 2.6 is fulfilled, and a straightforward computation shows that $K_{3}=89 / 3$, so assumption (b) in Corollary 2.6 is satisfied. Moreover, for any fixed

$$
\gamma_{0}>\log _{2 \sqrt{3} / 3} \frac{89}{6} \frac{e^{-\sqrt{3}}\left(e^{3}-e^{2}\right)}{e^{3 / 4}-e^{1 / 4}}
$$

we see that

$$
\frac{1}{2}(e-1) \sqrt{3}^{\gamma_{0}} e^{-\sqrt{3}}<\frac{3}{89}\left(e^{3 / 4}-e^{1 / 4}\right) 2^{\gamma_{0}} e^{-2} .
$$

Therefore, with any $\gamma \geq \gamma_{0}$ Assumption (c) in Corollary 2.6 is satisfied. In particular, since

$$
\limsup _{|t| \rightarrow+\infty} \frac{F(x, t)}{t^{2}}=0,
$$

Assumption (c) in Corollary 2.6 is satisfied. So, Corollary 2.6 is applicable to the boundary value problem

$$
\left\{\begin{array}{l}
-u^{\prime \prime}=\left[\lambda\left(u^{+}\right)^{\gamma-1} e^{x-u^{+}}\left(\gamma-u^{+}\right)+u^{+}\right]\left(2+\sin u^{\prime}\right)^{-1}, \\
u(0)=u(1)=0,
\end{array}\right.
$$

for each $\lambda \in] 89 e^{2} /\left(3\left(e^{3 / 4}-e^{1 / 4}\right) 2^{\gamma}\right), 2 e^{\sqrt{3}} /\left(\sqrt{3}^{\gamma}(e-1)\right)[$. 
Now, we consider a special situation of the results. Set $g(t)=0$ for all $t \in \mathbb{R}$. Then, we have the following consequences from Corollaries 2.6 and 2.7, respectively.

COROllary 2.9. Assume that there exist four positive constants $c, d, \mu$ and $\nu$ with $\mu+\nu<b-a$ such that the assumption (a) in Corollary 2.6 holds. Furthermore, suppose that:

(a) $\mu H\left(\frac{d}{\mu}\right)+\nu H\left(-\frac{d}{\nu}\right)>\frac{2 c^{2}}{M(b-a)}$,

(b) $\frac{M(b-a)}{2 c^{2}} \int_{a}^{b} \sup _{t \in[-c, c]} F(x, t)<\frac{\int_{a+\mu}^{b-\nu} F(x, d) d x}{\mu H\left(\frac{d}{\mu}\right)+\nu H\left(-\frac{d}{\nu}\right)}$,

(c) $\limsup _{|t| \rightarrow+\infty} \frac{F(x, t)}{t^{2}}<\frac{1}{(b-a) c^{2}} \int_{a}^{b} \sup _{t \in[-c, c]} F(x, t) d x$ uniformly with respect to $x \in[a, b]$.

Then, for each

$$
\left.\lambda \in \Lambda_{4}:=\right] \frac{\mu H\left(\frac{d}{\mu}\right)+\nu H\left(-\frac{d}{\nu}\right)}{\int_{a+\mu}^{b-\nu} F(x, d) d x}, \frac{2 c^{2}}{M(b-a) \int_{a}^{b} \sup _{t \in[-c, c]} F(x, t)}[
$$

the problem

$$
\left\{\begin{array}{l}
-u^{\prime \prime}=\lambda f(x, u) h\left(u^{\prime}\right) \quad \text { in }(a, b), \\
u(a)=u(b)=0
\end{array}\right.
$$

admits at least three distinct weak solutions in $W_{0}^{1,2}([a, b])$.

Corollary 2.10. Suppose that $f:[a, b] \times \mathbb{R} \rightarrow \mathbb{R}$ satisfies the condition $f(x, t) \geq 0$ for all $x \in[a, b]$ and $t \geq 0$. Assume that there exist five positive constants $c_{1}, c_{2}, d, \mu$ and $\nu$ with $\mu+\nu<b-a$ such that:
(a) $\frac{4 c_{1}^{2}}{M(b-a)}<\mu H\left(\frac{d}{\mu}\right)+\nu H\left(-\frac{d}{\nu}\right)<\frac{c_{2}^{2}}{M(b-a)}$,
(b) $\frac{M(b-a)}{2 c_{1}^{2}} \int_{a}^{b} \sup _{t \in\left[-c_{1}, c_{1}\right]} F(x, t) d x<\frac{2}{3} \frac{\int_{a+\mu}^{b-\nu} F(x, d) d x}{\mu H\left(\frac{d}{\mu}\right)+\nu H\left(-\frac{d}{\nu}\right)}$,
(c) $\frac{M(b-a)}{2 c_{2}^{2}} \int_{a}^{b} \sup _{t \in\left[-c_{2}, c_{2}\right]} F(x, t) d x<\frac{1}{3} \frac{\int_{a+\mu}^{b-\nu} F(x, d) d x}{\mu H\left(\frac{d}{\mu}\right)+\nu H\left(-\frac{d}{\nu}\right)}$. 
Then, for each

$$
\lambda \in] \frac{3}{2} \frac{\mu H\left(\frac{d}{\mu}\right)+\nu H\left(-\frac{d}{\nu}\right)}{\int_{a+\mu}^{b-\nu} F(x, d) d x}, \Theta_{4}[
$$

where

$$
\Theta_{4}:=\min \left\{\frac{\frac{2 c_{1}^{2}}{M(b-a)}}{\int_{a}^{b} \sup _{t \in\left[-c_{1}, c_{1}\right]} F(x, t) d x}, \frac{\frac{c_{2}^{2}}{M(b-a)}}{\int_{a}^{b} \sup _{t \in\left[-c_{2}, c_{2}\right]} F(x, t) d x}\right\}
$$

the problem (2.4) admits at least three non-negative weak solutions $v^{1}, v^{2}, v^{3}$ such that

$$
\left|v^{j}(x)\right|<c_{2} \quad \text { for each } x \in[a, b], j=1,2,3 \text {. }
$$

The following result is a consequence of Corollary 2.10.

COROLlary 2.11. Let $f: \mathbb{R} \rightarrow \mathbb{R}$ be a non-negative continuous function such that

$$
\lim _{t \rightarrow 0^{+}} \frac{f(t)}{t}=0, \quad \int_{0}^{6} f(\xi) d \xi<3 \int_{0}^{1} f(\xi) d \xi .
$$

Then, for every $\lambda \in] 12 / \int_{0}^{1} f(\xi) d \xi, 36 / \int_{0}^{6} f(\xi) d \xi[$ the problem

$$
\left\{\begin{array}{l}
-u^{\prime \prime}=\lambda f(u) \quad \text { in }(0,1), \\
u(0)=u(1)=0,
\end{array}\right.
$$

admits at least three non-negative classical solutions $v^{1}, v^{2}, v^{3}$ such that

$$
\left|v^{j}(x)\right|<6 \quad \text { for each } x \in[0,1], j=1,2,3 \text {. }
$$

Proof. Choose $a=0, b=1$. Set $f(x, t)=f(t)$ for all $x \in[0,1]$ and $t \in \mathbb{R}$, and $h \equiv 1$. Put $d=1$ and $c_{2}=6$. Therefore, by a simple computation we have

$$
\begin{aligned}
\frac{3}{2} \frac{}{\mu H\left(\frac{d}{\mu}\right)+\nu H\left(-\frac{d}{\nu}\right)} & =\frac{12}{\int_{a+\mu}^{b-\nu} F(x, d) d x}, \\
\frac{\frac{c_{2}^{2}}{M(b-a)}}{\int_{a}^{b} \sup _{t \in\left[-c_{2}, c_{2}\right]} F(x, t) d x} & =\frac{36}{\int_{0}^{6} f(\xi) d \xi} .
\end{aligned}
$$


Moreover, since $\lim _{t \rightarrow 0^{+}} f(t) / t=0$, there is a positive constant $c_{1}<1$ such that

$$
\frac{1}{c_{1}^{2}} \int_{0}^{c_{1}} f(\xi) d \xi<\frac{1}{6} \int_{0}^{1} f(\xi) d \xi \text { and } \quad \frac{c_{1}^{2}}{\int_{0}^{c_{1}} f(\xi) d \xi}<\frac{18}{\int_{0}^{6} f(\xi) d \xi}
$$

Hence, it is easy to see that all assumptions of Corollary 2.10 are satisfied, and the conclusion follows.

REMARK 2.12. We would like to stress that our results generalize previous works. Specifically, Corollaries 2.9 and 2.10 extend Theorem 1 in [25], separately in the setting $[a, b]=[0,1]$ and $2 c^{2} /(M(b-a))=r$. In this respect, it is worth remarking that the growth condition

$$
\int_{0}^{t} f(x, \xi) d \xi \leq \eta\left(1+|t|^{s}\right),
$$

with $\eta>0$ and $0<s<2$ assumed in Theorem 1 in [25], is stronger than our substitute assumptions. Taking $h=1$, Corollaries 2.9 and 2.10 extend Theorem 2 of Bonanno [7] and Theorem 1 of Candito [17].

REMARK 2.13. The weak solutions of the problem (1.1) where $f$ is continuous, by using standard methods, belong to $C^{2}([a, b])$. Namely, in this case, the classical and the weak solutions of the problem (1.1) coincide.

We end this section by proving Theorem 1.1.

Proof of Theaorem 1.1. Fix

$$
\lambda>\lambda^{*}:=\frac{H(4 d /(b-a))+H(-4 d /(b-a))}{2 F(d)} \text { for some } d>0
$$

where

$$
H(t)=\int_{0}^{t} \int_{0}^{\tau} \frac{1}{h(\delta)} d \delta d \tau
$$

for each $t \in \mathbb{R}$. Taking into account that $\liminf _{\xi \rightarrow 0} F(\xi) / \xi^{2}=0$, there is $\left\{c_{m}\right\}_{m \in \mathbb{N}} \subseteq$ ] $0,+\infty\left[\right.$ such that $\lim _{m \rightarrow+\infty} c_{m}=0$ and

$$
\lim _{m \rightarrow+\infty} \frac{\sup _{|\xi| \leq c_{m}} F(\xi)}{c_{m}^{2}}=0 .
$$

In fact, one has

$$
\lim _{m \rightarrow+\infty} \frac{\sup _{|\xi| \leq c_{m}} F(\xi)}{c_{m}^{2}}=\lim _{m \rightarrow+\infty} \frac{F\left(\xi_{c_{m}}\right)}{\xi_{c_{m}}^{2}} \cdot \frac{\xi_{c_{m}}^{2}}{c_{m}^{2}}=0,
$$


where $F\left(\xi_{c_{m}}\right)=\sup _{|\xi| \leq c} F(\xi)$. Hence, there is $\bar{c}>0$ such that

$$
\frac{\sup _{|\xi| \leq \bar{c}} F(\xi)}{\bar{c}^{2}}<\min \left\{\frac{4 F(d)}{M(b-a)^{2}\left(H\left(\frac{4 d}{b-a}\right)+H\left(-\frac{4 d}{b-a}\right)\right)} ; \frac{2}{\lambda M(b-a)^{2}}\right\}
$$

and

$$
\bar{c}<\frac{b-a}{2 \sqrt{2}} \sqrt{M\left(H\left(\frac{4 d}{b-a}\right)+H\left(-\frac{4 d}{b-a}\right)\right)}
$$

where $M:=\sup h$. From Corollary 2.9 the conclusion follows.

\section{Proofs of the main results}

Proof of Theorem 2.1. We proceed by applying Theorem 1.2 for $X=$ $W_{0}^{1,2}([a, b])$ equipped with the norm

$$
\|u\|=\left(\int_{a}^{b}\left|u^{\prime}(x)\right|^{2} d x\right)^{1 / 2}
$$

and the functionals $\Phi, \Psi: X \rightarrow \mathbb{R}$ given by

$$
\Phi(u)=\int_{a}^{b}\left[G(x, u(x))+H\left(x, u^{\prime}(x)\right)\right] d x
$$

and

$$
\Psi(u)=\int_{a}^{b} F(x, u(x)) d x
$$

for each $u \in X$. It is well known that $J$ is a Gâteaux differentiable functional and sequentially weakly lower semicontinuous whose Gâteaux derivative at the point $u \in X$ is the functional $\Psi^{\prime}(u) \in X^{*}$, given by

$$
\Psi^{\prime}(u)(v)=\int_{a}^{b} f(x, u(x)) v(x) d x
$$

for every $v \in X$. We claim that $\Psi^{\prime}: X \rightarrow X^{*}$ is a compact operator. Indeed, for fixed $u \in X$, assume $u_{n} \rightarrow u$ weakly in $X$ as $n \rightarrow+\infty$. Then $u_{n} \rightarrow u$ strongly in $C([a, b])$. Since $f(x, \cdot)$ is continuous in $\mathbb{R}$ for every $x \in[a, b]$, we get that $f\left(x, u_{n}\right) \rightarrow f(x, u)$ strongly as $n \rightarrow+\infty$. By the Lebesgue control convergence theorem, $\Psi^{\prime}\left(u_{n}\right) \rightarrow \Psi^{\prime}(u)$ strongly, which means that $\Psi^{\prime}$ is strongly continuous, then it is a compact operator. Hence the claim holds true. 
Moreover, $\Phi$ is a Gâteaux differentiable functional whose Gâteaux derivative at the point $u \in X$ is the functional $\Phi^{\prime}(u) \in X^{*}$, given by

$$
\begin{aligned}
\Phi^{\prime}(u)(v) & =\int_{a}^{b}\left[H^{\prime}\left(x, u^{\prime}(x)\right) v^{\prime}(x)-g(x, u(x)) v(x)\right] d x \\
& =\int_{a}^{b}\left[\left(\int_{0}^{u^{\prime}(x)} \frac{1}{h(x, \tau)} d \tau\right) v^{\prime}(x)-g(x, u(x)) v(x)\right] d x
\end{aligned}
$$

for every $v \in X$. Furthermore, $\Phi^{\prime}$ is a Lipschitzian operator. Indeed, for any $u, v \in X$, taking (2.1) into account since

$$
\max _{x \in[a, b]}|u(x)| \leq \frac{(b-a)^{1 / 2}}{2}\|u\|
$$

for each $u \in X$, it follows that

$$
\begin{aligned}
\left\|\Phi^{\prime}(u)-\Phi^{\prime}(v)\right\| X^{*} & =\sup _{\|w\| \leq 1}\left|\left\langle\Phi^{\prime}(u)-\Phi^{\prime}(v), w\right\rangle\right| \\
\leq & \sup _{\|w\| \leq 1} \int_{a}^{b}\left|\int_{u^{\prime}(x)}^{v^{\prime}(x)} \frac{1}{h(x, \tau)} d \tau \| w^{\prime}(x)\right| d x \\
& +\sup _{\|w\| \leq 1} \int_{a}^{b}|g(x, u(x))-g(x, v(x)) \| w(x)| d x \\
\leq & \left(\frac{1}{m}+\frac{L}{4}(b-a)^{2}\right)\|u-v\| .
\end{aligned}
$$

In particular, $\Phi$ is continuously Gâteaux differentiable. Bearing (2.1) in mind, and using (3.3), we obtain

$$
\begin{aligned}
\left\langle\Phi^{\prime}(u)-\Phi^{\prime}(v), u-v\right\rangle= & \int_{a}^{b}\left(\int_{v^{\prime}(x)}^{u^{\prime}(x)} \frac{1}{h(x, \tau)} d \tau\right)\left(u^{\prime}(x)-v^{\prime}(x)\right) d x \\
& -\int_{a}^{b}(g(x, u(x))-g(x, v(x)))(u(x)-v(x)) d x \\
\geq & \left(\frac{1}{M}-\frac{L}{4}(b-a)^{2}\right)\|u-v\|^{2}
\end{aligned}
$$

for $u, v \in X$. Due to the assumption $L M(b-a)^{2}<4$, it follows that $\Phi^{\prime}$ is a strongly monotone operator, so $\Phi^{\prime}$ is uniformly monotone and we obtain $\left(\Phi^{\prime}\right)^{-1}: X^{*} \rightarrow X$ exists and is continuous. Using again that $\Phi^{\prime}$ is uniformly monotone, we get that $\Phi$ is convex and continuous, and so is sequentially weakly lower semicontinuous.

Choose $u_{1}=w$, from (3.1) and assumption (a) in Theorem 2.1, we obtain $0<r<\Phi\left(u_{1}\right)$. Moreover, since $h$ is bounded away from zero and $g$ is continuous and satisfies $(2.1)$ with $g(\cdot, 0)=0$, from (3.1) we see that

$$
\Phi(u) \geq \frac{1}{2}\left(\frac{1}{M}-\frac{L}{4}(b-a)^{2}\right)\|u\|^{2} \quad \text { for all } u \in X,
$$


which yields

$$
\begin{aligned}
\left.\left.\Phi^{-1}(]-\infty, r\right]\right) & =\{u \in X: \Phi(u) \leq r\} \\
& =\left\{u \in X: \frac{1}{2}\left(\frac{1}{M}-\frac{L}{4}(b-a)^{2}\right)\|u\|^{2} \leq r\right\} \\
& \subseteq\left\{u \in X:|u(x)| \leq \sqrt{\frac{2 M(b-a) r}{4-L M(b-a)^{2}}} \text { for all } x \in[a, b]\right\},
\end{aligned}
$$

that leads to

$$
\begin{aligned}
\sup _{\left.\left.u \in \Phi^{-1}(]-\infty, r\right]\right)} \Psi(u) & =\sup _{\left.\left.u \in \Phi^{-1}(]-\infty, r\right]\right)} \int_{a}^{b} F(x, u(x)) d x \\
& \leq \int_{a}^{b} \sup _{t \in\left[-\sqrt{\frac{2 M(b-a) r}{4-L M(b-a)^{2}}}, \sqrt{\frac{2 M(b-a) r}{4-L M(b-a)^{2}}}\right]} F(x, t) d x .
\end{aligned}
$$

Therefore, assumption (b) in Theorem 2.1 implies that

$$
\begin{aligned}
\sup _{\left.\left.u \in \Phi^{-1}(]-\infty, r\right]\right)} \Psi(u) & \leq \int_{a}^{b} \sup _{t \in\left[-\sqrt{\frac{2 M(b-a) r}{4-L M(b-a)^{2}}}, \sqrt{\frac{2 M(b-a) r}{4-L M(b-a)^{2}}}\right]} F(x, t) d x \\
& <r \frac{\int_{a}^{b} F(x, w(x)) d x}{\int_{a}^{b}\left[G(x, w(x))+H\left(x, w^{\prime}(x)\right)\right] d x}=r \frac{\Psi(w)}{\Phi(w)},
\end{aligned}
$$

namely, assumption (a) of Theorem 1.2 is fulfilled.

Furthermore, due to assumption (c) in Theorem 2.1, there exist two constants $\gamma, \eta \in \mathbb{R}$ with

$$
\gamma<\frac{1}{r} \int_{a}^{b} \sup _{t \in\left[-\sqrt{\frac{2 M(b-a) r}{4-L M(b-a)^{2}}}, \sqrt{\frac{2 M(b-a) r}{4-L M(b-a)^{2}}}\right]} F(x, t) d x
$$

such that

$$
\frac{2 M(b-a)^{2}}{4-L M(b-a)^{2}} F(x, t) \leq \gamma t^{2}+\eta \quad \text { for a.e. } x \in[a, b] .
$$

Fix $u \in X$. Then

$$
F(x, u(x)) \leq \frac{4-L M(b-a)^{2}}{2 M(b-a)^{2}}\left(\gamma|u(x)|^{2}+\eta\right) \quad \text { for a.e. } x \in[a, b]
$$


Now, in order to prove the coercivity of the functional $\Phi-\lambda \Psi$, first we assume that $\gamma>0$. So, for any fixed $\lambda \in \Lambda_{1}$, from (3.1)-(3.5), we have

$$
\begin{aligned}
& \Phi(u)-\lambda \Psi(u)=\int_{a}^{b}\left[G(x, u(x))+H\left(x, u^{\prime}(x)\right)\right] d x-\lambda \int_{a}^{b} F(x, u(x)) d x \\
& \geq \frac{1}{2}\left(\frac{1}{M}-\frac{L}{4}(b-a)^{2}\right)\|u\|^{2} \\
& -\lambda \gamma \frac{4-L M(b-a)^{2}}{2 M(b-a)^{2}} \int_{a}^{b}|u(x)|^{2} d x-\frac{4-L M(b-a)^{2}}{2 M(b-a)} \lambda \eta \\
& \geq \frac{1}{2}\left(\frac{1}{M}-\frac{L}{4}(b-a)^{2}\right)\|u\|^{2} \\
& -\lambda \gamma \frac{4-L M(b-a)^{2}}{2 M(b-a)^{2}} \frac{(b-a)^{2}}{4}\|u\|^{2}-\frac{4-L M(b-a)^{2}}{2 M(b-a)} \lambda \eta \\
& =\frac{4-L M(b-a)^{2}}{8 M}(1-\lambda \gamma)\|u\|^{2}-\frac{4-L M(b-a)^{2}}{2 M(b-a)} \lambda \eta \\
& \geq \frac{4-L M(b-a)^{2}}{8 M} \\
& \cdot\left(1-\gamma r / \int_{a}^{b} \sup _{t \in\left[-\sqrt{\frac{2 M(b-a) r}{4-L M(b-a)^{2}}}, \sqrt{\frac{2 M(b-a) r}{4-L M(b-a)^{2}}}\right]} F(x, t) d x\right)\|u\|^{2} \\
& -\frac{4-L M(b-a)^{2}}{2 M(b-a)} \frac{r \eta}{\int_{a}^{b} \sup _{t \in\left[-\sqrt{\frac{2 M(b-a) r}{4-L M(b-a)^{2}}}, \sqrt{\frac{2 M(b-a) r}{4-L M(b-a)^{2}}}\right]} F(x, t) d x},
\end{aligned}
$$

and thus

$$
\lim _{\|u\| \rightarrow+\infty}(\Phi(u)-\lambda \Psi(u))=+\infty,
$$

which means that the functional $\Phi-\lambda \Psi$ is coercive. On the other hand, if $\gamma \leq 0$, Clearly, we get $\lim _{\|u\| \rightarrow+\infty}(\Phi(u)-\lambda \Psi(u))=+\infty$. Both cases lead to the coercivity of functional $\Phi-\lambda \Psi$. So, assumption (b) of Theorem 1.2 is satisfied. Now, we can apply Theorem 1.2. Hence, by using Theorem 1.2, taking into account that the weak solutions of (1.1) are exactly the solutions of the equation $\Phi^{\prime}(u)-\lambda \Psi^{\prime}(u)=0$, the problem (1.1) admits at least three distinct weak solutions.

Proof of Theorem 2.2. Let $X, \Phi$ and $\Psi$ be as in the proof of Theorem 2.1. Let us apply Theorem 1.3 to our functionals. Obviously, $\Phi$ and $\Psi$ satisfy the condition (a) of Theorem 1.3.

Now, we show that the functional $\Phi-\lambda \Psi$ satisfies the Assumption (b) of Theorem 1.3. Let $u^{*}$ and $u^{\star \star}$ be two local minima for $\Phi-\lambda \Psi$. Then $u^{*}$ and 
$u^{\star \star}$ are critical points for $\Phi-\lambda \Psi$, and so, they are weak solutions for the problem (1.1). Since $f(x, t) \geq 0$ for all $(x, t) \in[a, b] \times\left(\mathbb{R}^{+} \cup\{0\}\right)$, from the Weak Maximum Principle (see for instance [16]) we deduce $u^{\star}(x) \geq 0$ and $u^{\star \star}(x) \geq 0$ for every $x \in[a, b]$. So, it follows that $s u^{\star}+(1-s) u^{\star \star} \geq 0$ for all $s \in[0,1]$, and that $f\left(x, s u^{\star}+(1-s) u^{\star \star}\right) \geq 0$, and consequently, $\Psi\left(s u^{\star}+(1-s) u^{\star \star}\right) \geq 0$ for all $s \in[0,1]$. Moreover, from the condition

$$
2 r_{1}<\int_{a}^{b}\left[G(x, u(x))+H\left(x, u^{\prime}(x)\right)\right] d x<\frac{r_{2}}{2},
$$

we observe $2 r_{1}<\Phi(w)<r_{2} / 2$. Note that

$$
\Phi^{-1}(]-\infty, r_{1}[)=\left\{u \in X:|u(x)|<\sqrt{\frac{2 M(b-a) r_{1}}{4-L M(b-a)^{2}}} \text { for all } x \in[a, b]\right\},
$$

we have

$$
\begin{aligned}
\sup _{u \in \Phi^{-1}(]-\infty, r_{1}[)} \Psi(u) & =\sup _{u \in \Phi^{-1}(]-\infty, r_{1}[)} \int_{a}^{b} F(x, u(x)) d x \\
& \leq \int_{a}^{b} \sup _{t \in\left[-\sqrt{\frac{2 M(b-a) r_{1}}{4-L M(b-a)^{2}}}, \sqrt{\frac{2 M(b-a) r_{1}}{4-L M(b-a)^{2}}}\right]} F(x, t) d x .
\end{aligned}
$$

Therefore, owing to the assumption (a) of Theorem 2.2, we infer

$$
\begin{aligned}
\frac{1}{r_{1}} \sup _{u \in \Phi^{-1}(]-\infty, r_{1}[)} \Psi(u)=\frac{1}{r_{1}} \sup _{u \in \Phi^{-1}(]-\infty, r_{1}[)} \int_{a}^{b} F(x, u(x)) d x & \int_{a}^{b} F(x, t) d x \\
\leq & \frac{\left.\sup _{t \in\left[-\sqrt{\frac{2 M(b-a) r_{1}}{4-L M(b-a)^{2}}}\right.}, \sqrt{\frac{2 M(b-a) r_{1}}{4-L M(b-a)^{2}}}\right]}{\int_{a}^{b}\left[G(x, u(x))+H\left(x, u^{\prime}(x)\right)\right] d x}=\frac{2}{3} \frac{\Psi(w)}{\Phi(w)} .
\end{aligned}
$$

As above, bearing the assumption (b) of Theorem 2.2 in mind, we deduce

$$
\begin{aligned}
\frac{1}{r_{2}} \sup _{u \in \Phi^{-1}(]-\infty, r_{2}[)} \Psi(u) & =\frac{1}{r_{2}} \sup _{u \in \Phi^{-1}(]-\infty, r_{2}[)} \int_{a}^{b} F(x, u(x)) d x \\
& \leq \frac{1}{r_{2}} \int_{a}^{b} \underset{t \in\left[-\sqrt{\frac{2 M(b-a) r_{2}}{4-L M(b-a)^{2}}}, \sqrt{\frac{2 M(b-a) r_{2}}{4-L M(b-a)^{2}}}\right]}{ } F(x, t) d x \\
& <\frac{1}{3} \frac{\int_{a}^{b} F(x, w(x)) d x}{\int_{a}^{b}\left[G(x, u(x))+H\left(x, u^{\prime}(x)\right)\right] d x}=\frac{2}{3} \frac{\Psi(w)}{\Phi(w)}
\end{aligned}
$$

So, the assumptions (1.2) and (1.3) in Theorem 1.3 are satisfied. Hence, by using Theorem 1.3, taking into account that the weak solutions of the problem (1.1) 
are exactly the solutions of the equation $\Phi^{\prime}(u)-\lambda \Psi^{\prime}(u)=0$, the problem (1.1) admits at least three distinct weak solutions in $X$.

Acknowledgements. The authors express their gratitude to professor Gabriele Bonanno for his helpful remarks, which greatly improved the results of this paper. Research of Shapour Heidarkhani was in part supported by a grant from IPM (No. 91470046).

\section{REFERENCES}

[1] G.A. Afrouzi And S. Heidarkhani, Three solutions for a quasilinear boundary value problem, Nonlinear Anal. 69 (2008), 3330-3336.

[2] R.P. Agarwal, H.B. Thompson and C.C. Tisdell, On the existence of multiple solutions to boundary value problems for second order ordinary differential equations, Dynam. Systems Appl. 16 (2007), 595-609.

[3] D. Averna and G. Bonanno, A mountain pass theorem for a suitable class of functions, Rocky Mountain J. Math. 39 (2009), 707-727.

[4] A three critical points theorem and its applications to the ordinary Dirichlet problem, Topol. Methods Nonlinear Anal. 22 (2003), 93-103.

[5] R.I. Avery and J. Henderson, Three symmetric positive solutions for a second-order boundary value problem, Appl. Math. Lett. 13 (2000), 1-7.

[6] G. Bonanno, A critical points theorem and nonlinear differential problems, J. Global Optimization 28 (2004), 249-258.

[7] Existence of three solutions for a two point boundary value problem, Appl. Math. Lett. 13 (2000), 53-57.

[8] G. Bonanno and P. Candito, Non-differentiable functionals and applications to elliptic problems with discontinuous nonlinearities, J. Differential Equations 244 (2008), 3031-3059.

[9] G. Bonanno And A. Chinnì, Existence of three solutions for a perturbed two-point boundary value problem, Appl. Math. Lett. 23 (2010), 807-811.

[10] G. Bonanno And G. D'Aguì, A Neumann boundary value problem for the SturmLiouville equation, Appl. Math. Comput. 15 (2009), 318-327.

[11] Multiplicity results for a perturbed elliptic Neumann problem, Article ID 564363, Abstr. Appl. Anal. 2010 (2010), 10 p.

[12] G. Bonanno, S. Heidarkhani and D. O'Regan, Multiple solutions for a class of Dirichlet quasilinear elliptic systems driven by a $(p, q)$-Laplacian operator, Dynam. Systems Appl. 20 (2011), 89-100.

[13] G. Bonanno and S.A. Marano, On the structure of the critical set of non-differentiable functionals with a weak compactness condition, Appl. Anal. 89 (2010), 1-10.

[14] G. Bonanno, G. Molica Bisci And V. Rădulescu, Infinitely many solutions for a class of nonlinear eigenvalue problem in Orlicz-Sobolev spaces, C.R. Acad. Sci. Paris Sér. I 349 (2011), 263-268.

[15] G. Bonanno nd G. Riccobono, Multiplicity results for Sturm-Liouville bondary value problems, Appl. Math. Comput. 210 (2009), 294-297.

[16] H. BrÉzIs, Analyse Functionelle - Théorie et Applications, Masson, Paris, 1983.

[17] P. CAndito, Existence of three solutions for a nonautonomous two point boundary value problem, J. Math. Anal. Appl. 252 (2000), 532-537. 
[18] G. D'Aguì And G. MolicA BisCI, Three non-zero solutions for elliptic Neumann problems, Anal. Appl. 9 (2011), 383-394.

[19] M. Ghergu And V. Rădulescu, Singular Elliptic Problems. Bifurcation and Asymptotic Analysis, Oxford Lecture Series in Mathematics and Its Applications, vol. 37, Oxford University Press, 2008.

[20] J.R. Graef, S. Heidarkhani And L. Kong, A critical points approach for the existence of multiple solutions of a Dirichlet quasilinear system, J. Math. Anal. Appl. 388 (2012), $1268-1278$.

[21] S. Heidarkhani and D. Motreanu, Multiplicity results for a two-point boundary value problem, Panamer. Math. J. 19/3 (2009), 69-78.

[22] J. Henderson and H.B. Thompson, Existence of multiple solutions for second order boundary value problems, J. Differential Equations 166 (2000), 443-454.

[23] Multiple symmetric positive solutions for a second order boundary value problem, Proc. Amer. Math. Soc. 128 (2000), 2373-2379.

[24] A. Kristály, V. Rădulescu and C. Varga, Variational Principles in Mathematical Physics, Geometry, and Economics: Qualitative Analysis of Nonlinear Equations and Unilateral Problems, Encyclopedia of Mathematics and its Applications, vol. 136, Cambridge University Press, Cambridge, 2010.

[25] R. Livrea, Existence of three solutions for a quasilinear two point boundary value problem, Arch. Math. 79 (2002), 288-298.

[26] S.A. Marano and D. Motreanu, On a three critical points theorem for non-differentiable functions and applications nonlinear boundary value problems, Nonlinear Anal. 48 (2002), 37-52.

[27] B. RiCCERI, A three critical points theorem revisited, Nonlinear Anal. 70 (2009), 30843089.

[28] - On a three critical points theorem, Arch. Math. (Basel) 75 (2000), 220-226.

[29] Existence of three solutions for a class of elliptic eigenvalue problem, Math. Comput. Modelling 32 (2000), 1485-1494.

[30] E. Zeidler, Nonlinear Functional Analysis and its Applications, vol. II, Springer, Berlin, Heidelberg, New York, 1985.

Shapour HeidarkHani

Department of Mathematics

Faculty of Sciences

Razi University

67149 Kermanshah, IRAN

and

School of Mathematics

Institute for Research in Fundamental Sciences (IPM)

P.O. Box 19395-5746

Tehran, IRAN

E-mail address: address s.heidarkhani@razi.ac.ir

JoHnNy HENDERSON

Department of Mathematics

Baylor University

Waco, TX 76798-7328, USA

E-mail address: Johnny_Henderson@baylor.edu

TMNA : Volume $44-2014-\mathrm{N}^{\mathrm{o}} 1$ 